\title{
Comorbidities and elevated IL-6 associate with negative outcome in adult-onset asthma
}

\author{
Pinja Ilmarinen¹, Leena E. Tuomisto', Onni Niemelä², Joanna Danielsson², \\ Jussi Haanpää ${ }^{3}$, Terhi Kankaanranta ${ }^{4}$ and Hannu Kankaanranta ${ }^{1,5}$
}

Affiliations: ${ }^{1}$ Dept of Respiratory Medicine, Seinäjoki Central Hospital, Seinäjoki, Finland. ${ }^{2}$ Dept of Laboratory Medicine, Seinäjoki Central Hospital, Seinäjoki, and University of Tampere, Tampere, Finland. ${ }^{3}$ Dept of Clinical Physiology, Seinäjoki Central Hospital, Seinäjoki, Finland. ${ }^{4}$ Police University College, Tampere, Finland. ${ }^{5}$ Dept of Respiratory Medicine, University of Tampere, Tampere, Finland.

Correspondence: Pinja Ilmarinen, Dept of Respiratory Medicine, Seinäjoki Central Hospital, FIN-60220 Seinäjoki, Finland. E-mail: pinja.ilmarinendepshp.fi

ABSTRACT The effect of systemic inflammation and comorbidities on treatment and outcome of adultonset asthma remains unknown and is the objective of this study.

As part of the Seinäjoki Adult Asthma Study (SAAS) with a 12-year follow-up, serum interleukin (IL)-6, high-sensitivity C-reactive protein (hsCRP) and lung function were measured and clinical information on comorbidities and medication collected from 170 patients with adult-onset asthma without chronic obstructive pulmonary disease.

At follow-up visit, $54 \%$ of the patients had systemic inflammation as indicated by elevated IL- 6 or hsCRP, 58\% had at least one comorbidity and 30\% at least two comorbidities (other than asthma related). Patients with systemic inflammation were treated with higher dose of inhaled corticosteroid (ICS) and they had lower lung function and higher blood neutrophils compared with patients without. Patients having $\geqslant 2$ comorbidities had lower Asthma Control Test score and this association remained significant in adjusted analysis. Patients with both systemic inflammation and comorbidities showed poorest outcome of asthma. In multivariate regression analysis, high ICS dose was predicted by elevated IL-6, elevated blood neutrophils and eosinophils and poorer lung function at baseline and follow-up.

Altogether, in patients with adult-onset asthma, elevated IL-6 was associated with use of high-dose ICS while multi-morbidity was linked to worse symptoms of asthma.

@ERSpublications

In adult-onset asthma elevated IL-6 is linked to high ICS dose and multimorbidity to worse symptoms of asthma http://ow.ly/IUtG3010z3v

Editorial comment in Eur Respir J 2016; 48: 979-981.

This article has supplementary material available from erj.ersjournals.com

Received: Dec 282015 | Accepted after revision: May 282016 | First published online: Aug 182016

This study is registered at ClinicalTrials.gov with identifier number NCT02733016.

Support statement: This study was funded by the Finnish Anti-Tuberculosis Association Foundation, Jalmari and Rauha Ahokas Foundation, Tampere Tuberculosis Foundation, the Competitive State Research Financing of the Expert Responsibility Area of Tampere University Hospital (Tampere, Finland) and the Medical Research Fund of Seinäjoki Central Hospital (Seinäjoki, Finland). Funding information for this article has been deposited with the Open Funder Registry.

Conflict of interest: Disclosures can be found alongside this article at erj.ersjournals.com

Copyright CERS 2016 


\section{Introduction}

Asthma with onset during adulthood has been suggested to be a distinctive phenotype of asthma. Adult-onset disease differs from childhood-onset asthma in many aspects. Childhood-onset asthma is characterised by male-predominance, familial atopy, T-helper 2 (Th2) cell predominant inflammation, good responsiveness to glucocorticoids and good prognosis $[1,2]$. In contrast, patients with adult-onset asthma are most often non-atopic females with poor prognosis and without a family history of asthma/atopy [2-5].

Patients with asthma often suffer from conditions such as allergic or non-allergic rhinitis, respiratory infections and gastro-oesophageal reflux. Chronic obstructive pulmonary disease (COPD) is recognised as a common comorbidity of asthma often developing in smoking asthmatics. In addition, adult patients with asthma have higher rates of comorbidities such as psychiatric diseases, diabetes mellitus and cardiovascular disease compared with the general population, and often have more than one comorbidity $[6,7]$. Asthmatics with comorbidities have high non-asthma-related healthcare use, being highest in those with active asthma [6] suggesting a link between asthma and comorbidities. Asthma with more than one comorbidity has been shown to result in decreased asthma-related quality of life [8] and a concomitant depressive disorder has been associated with poorer asthma control $[9,10]$.

Systemic inflammation, characterised by elevated levels of interleukin (IL)-6 and C-reactive protein (CRP), possesses a possible link between asthma and comorbidities. It is associated with diseases such as diabetes, cardiovascular disease, systemic rheumatic diseases and mental disorders in addition to asthma [11-13]. Even though obesity is the best known inducer of low-grade systemic inflammation, systemic inflammation has wider aetiology than obesity: it is enhanced for example by ageing, depression, stress and smoking [7, 14-16]. Overspill of inflammation from one organ has also been suggested as a possible origin of systemic inflammation [15] and is one suggested mechanism for comorbidities to affect asthma or vice versa. Alternatively, psychological stress of multi-morbidity may have a negative impact on asthma, for example via stress-related increase in systemic inflammation [14]. Association between elevated IL-6 and/or high sensitivity (hs) CRP, neutrophilic inflammation and worse lung function or control of asthma has been previously proposed $[15,16]$.

A patient with adult-onset asthma is typically a middle-aged woman with overweight. Therefore we hypothesised that systemic inflammation and comorbidities are common in patients with adult-onset disease and affect outcome and treatment of asthma in these patients. We aimed to study whether comorbidities and systemic inflammation together and separately affect the treatment and outcome of asthma.

\section{Methods}

\section{Study design and patients}

The current study was part of Seinäjoki Adult Asthma Study (SAAS). SAAS is a prospective, single-centre (Seinäjoki Central Hospital, Seinäjoki, Finland) 12-year follow-up study of a cohort of patients having new-onset asthma that was diagnosed at adult age ( $\geqslant 15$ years). Institutional permissions (TU1114 and LET) were obtained and the participants gave written informed consent to the study protocol approved by the ethics committee of Tampere University Hospital, Tampere, Finland (R12122). The protocol of SAAS-study has been published separately [17], and the inclusion and exclusion criteria are shown in table S1. Shortly, asthma was diagnosed by a respiratory physician during 1999-2002 based on typical symptoms and confirmed by objective lung function measurements [17]. After diagnosis, the patients were treated and monitored by their own treating physician either in the specialised care or in primary care. The total cohort consisted of 257 patients and 203 patients returned to the follow-up visit 12 years (mean 12.2 years, range 10.8-13.9 years) after diagnosis. At follow-up visit, asthma status and control, comorbidities and medication were evaluated using structured questionnaires and lung function and serum IL-6 and hsCRP were measured. Patients with suspected or co-existing COPD (post-bronchodilator forced expiratory volume in $1 \mathrm{~s}$ (FEV1)/forced vital capacity (FVC) $<0.7$ and $>10$ smoked pack years) were excluded from the main analyses (figure 1) because the main focus was to concentrate on the effects of less well known comorbidities. However, for some analyses, those patients who could be classified as having asthma-COPD overlap syndrome (ACOS) [18] were included as a separate group for comparison.

\section{Lung function, inflammatory parameters and other clinical measurements}

Lung function measurements were performed with a spirometer according to international recommendations [19]. More detailed information can be found in the online supplement. Fraction of exhaled nitric oxide ( $F$ eNO) was measured with a portable rapid-response chemiluminescent analyser according to American Thoracic Society standards [20] (flow rate $50 \mathrm{~mL} \cdot \mathrm{s}^{-1}$; NIOX System, Aerocrine, Solna, Sweden). Venous blood was collected and white blood cells differential counts were determined. Total immunoglobulin (Ig)E levels were measured by using ImmunoCAP (Thermo Scientific, Uppsala, Sweden). Laboratory assays were performed in an accredited laboratory (SFS-EN ISO/IEC 17025:2005 and ISO 15189:2007) of Seinäjoki 


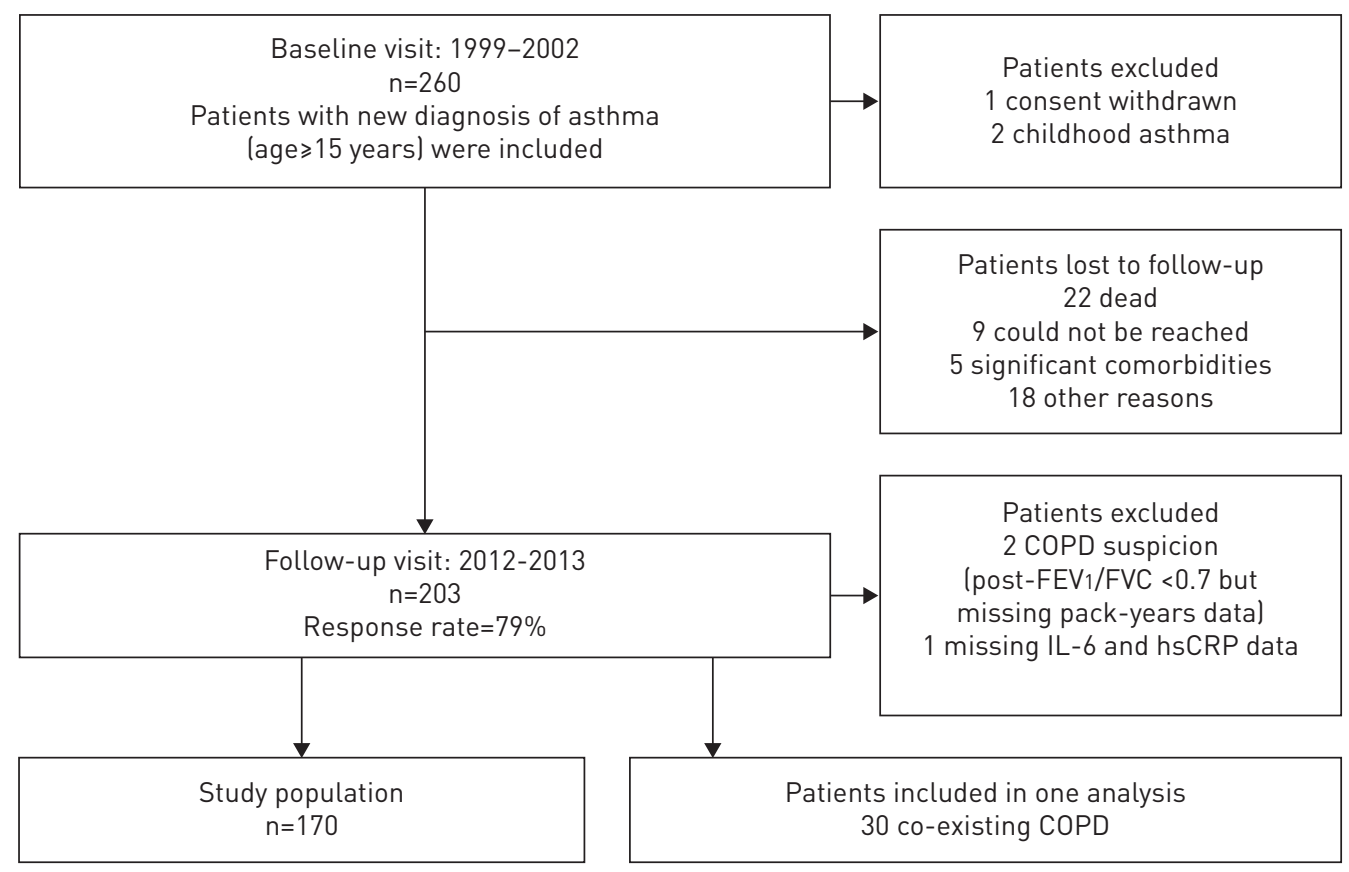

FIGURE 1 Flow chart of the study. COPD: chronic obstructive pulmonary disease; FEV1: forced expiratory volume in $1 \mathrm{~s}$; FVC: forced vital capacity; CRP: C-reactive protein.

Central Hospital. Patients completed Airways Questionnaire 20 (AQ20) [21] and Asthma Control Test (ACT). Assessment of asthma control was performed according to the Global Initiative for Asthma (GINA) 2010 report [22].

\section{Comorbidities}

Definition of comorbidities and their classification was based on a previous study [23]. Comorbidities were self-reported or based on self-reported medication. Unclear cases were confirmed from patient records. The conditions included as comorbidities are listed in the online supplement.

\section{Systemic inflammation}

Serum levels of IL-6 were determined by ELISA (R \& D Systems, Minneapolis, MN, USA) and hsCRP was measured using particle-enhanced immunoturbidometric method on Roche Cobas 8000 automated clinical chemistry analyser (Roche Diagnostics, Basel, Switzerland). The lower limits of detection were $0.7 \mathrm{pg} \cdot \mathrm{mL}^{-1}$ for IL- 6 and $0.3 \mathrm{mg} \cdot \mathrm{L}^{-1}$ for the hsCRP assay. Cut-off values used to define systemic inflammation (IL-6 level $>1.55 \mathrm{pg} \cdot \mathrm{mL}^{-1}$ and/or hsCRP $>4.12 \mathrm{mg} \cdot \mathrm{L}^{-1}$ ) were set based on upper quartile of healthy subjects [15].

\section{Statistical analysis}

Continuous data are expressed as mean $\pm \mathrm{SD}$ or median (interquartile range). Comparison between two groups was performed by using a t-test, Mann-Whitney rank sum test or Chi-squared-test. To analyse differences between more than two groups, a one-way ANOVA with Tukey's post hoc test, Kruskal-Wallis test or Chi-squared-test was carried out.

A multinomial logistic regression was performed when analysing predictors of ICS dose and binary logistic regression to predict poor ACT score. The correlation matrix was analysed and the explanatory variables not strongly correlated $(\mathrm{R}<0.7)$ were included in the analysis. In the model predicting high ICS dose, the predictors were selected based on univariate analysis and the combination of variables giving the best pseudo- $\mathrm{R}^{2}$ (Nagelkerke) chosen by using the enter method. Also forward and backward methods were carried out to aid selection of the best model. Statistical analyses were performed using SPSS software, version 22 (IBM SPSS, Chigaco, IL, USA). A p-value $<0.05$ was regarded as statistically significant.

\section{Results}

\section{Patient characteristics}

Characteristics of patients with adult-onset asthma at follow-up are shown in table 1 . In this cohort, 57.6\% had at least one comorbidity, $30 \%$ had at least two comorbidities, $52.4 \%$ showed elevated level of IL-6 $\left(>1.55 \mathrm{pg} \cdot \mathrm{mL}^{-1}\right)$ and $15.3 \%$ of the patients showed elevated level of hsCRP $\left(>4.12 \mathrm{mg} \cdot \mathrm{L}^{-1}\right)$. In total, $54.1 \%$ 
TABLE 1 Patient characteristics at follow-up visit

Total cohort

\begin{tabular}{|c|c|}
\hline Patients & 170 \\
\hline Male sex & $63(37.1)$ \\
\hline Age years & $57.2 \pm 14.0$ \\
\hline BMI $\mathrm{kg} \cdot \mathrm{m}^{-2}$ & $28.7 \pm 5.8$ \\
\hline Current/ex-smokers & $21(12.4) / 53(31.2)$ \\
\hline Pack years of smokers & $10(4-20)$ \\
\hline Uncontrolled asthma & $41(24.1)$ \\
\hline Daily ICS users & $132(77.6)$ \\
\hline ICS daily dose $\mu \mathrm{g}$ budesonide equivalents & $800(400-1000)$ \\
\hline Daily LABA & 78 (45.9) \\
\hline Daily LTRA & $24(14.1)$ \\
\hline Daily theophylline & $4(2.4)$ \\
\hline Pre-bronchodilator FEV $1 \%$ pred & $88 \pm 16$ \\
\hline Pre-bronchodilator FVC \% pred & $97 \pm 15$ \\
\hline Pre-bronchodilator FEV 1 /FVC ratio & $0.74 \pm 0.09$ \\
\hline Post-bronchodilator FEV $1 \%$ pred & $92 \pm 16$ \\
\hline Post-bronchodilator FVC \% pred & $98 \pm 15$ \\
\hline Post-bronchodilator FEV 1 /FVC ratio & $0.76 \pm 0.08$ \\
\hline Pre-bronchodilator FEV $1 \mathrm{~L}$ & $2.78 \pm 0.79$ \\
\hline Pre-bronchodilator FVC L & $3.78 \pm 1.00$ \\
\hline Post-bronchodilator FEV 1 L & $2.88 \pm 0.81$ \\
\hline Post-bronchodilator FVC L & $3.80 \pm 1.00$ \\
\hline $\mathrm{IL}-6 \mathrm{pg} \cdot \mathrm{mL}^{-1}$ & $1.71(1.12-2.70)$ \\
\hline hsCRP $\mathrm{mg} \cdot \mathrm{L}^{-1}$ & $1.23(0.53-2.53)$ \\
\hline AQ20 score & $4(2-7)$ \\
\hline ACT score & $22(19-24)$ \\
\hline
\end{tabular}

Data are presented as $\mathrm{n}(\%)$, mean \pm SD or median (interquartile range), unless otherwise stated. BMI: body mass index; ICS: inhaled corticosteroid; LABA: long-acting $\beta_{2}$-agonist; LTRA: leukotriene receptor antagonist; FEV1: forced expiratory volume in $1 \mathrm{~s}$; FVC: forced vital capacity; IL: interleukin; hsCRP: high-sensitivity C-reactive protein; AQ20: airways questionnaire 20; ACT: asthma control test.

had systemic inflammation (IL-6 and/or hsCRP elevated). Specific comorbidities of the patients are shown in figure 2.

Outcome of asthma in patients with or without systemic inflammation and comorbidity

We divided patients into four groups based on whether they had comorbidities, systemic inflammation or both. The largest group composed of patients with overlap of systemic inflammation and at least one comorbidity (38.2\%). The patients with comorbidities and systemic inflammation were older, had higher body mass index (BMI) and higher amount of smoked pack-years (table 2). They were also treated with higher dose of ICS, had worse lung function, higher AQ20 score, lower ACT score and higher blood neutrophils (table 2). Patients with systemic inflammation but no comorbidity used higher doses of ICS, whereas patients having $\geqslant 1$ comorbidity without systemic inflammation showed higher AQ20 score compared with the group lacking both.

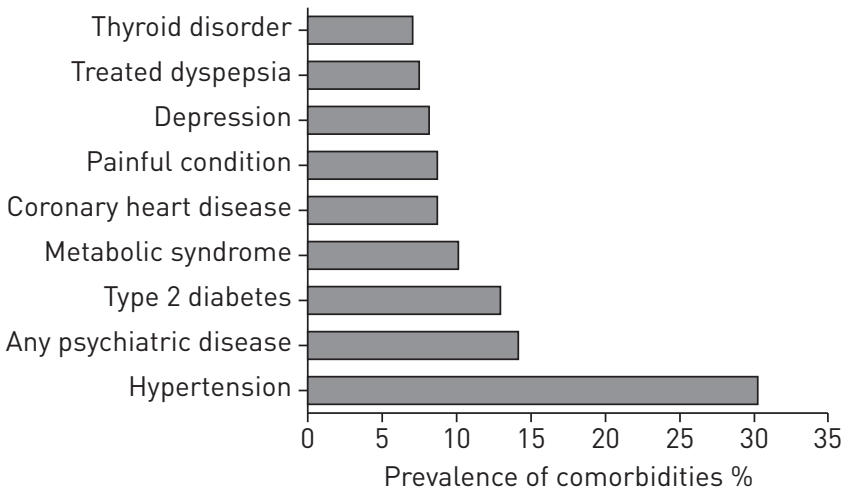


TABLE 2 Characteristics of patients with adult-onset asthma with and without systemic inflammation and comorbidities

\begin{tabular}{|c|c|c|c|c|}
\hline $\begin{array}{l}\text { No comorbidity and no } \\
\text { systemic inflammation }\end{array}$ & $\begin{array}{l}\geqslant 1 \text { comorbidity no } \\
\text { systemic inflammation }\end{array}$ & $\begin{array}{l}\text { No comorbidity and } \\
\text { systemic inflammation }\end{array}$ & $\begin{array}{l}\geqslant 1 \text { comorbidity and } \\
\text { systemic inflammation }\end{array}$ & $\mathrm{p}$-value \\
\hline 45 & 33 & 27 & 65 & \\
\hline $19(42.2)$ & $10(30.3)$ & $8(29.6)$ & $26(40.0)$ & 0.564 \\
\hline $49.26 \pm 14.76$ & $58.05 \pm 10.59 *$ & $54.77 \pm 12.79$ & $63.19 \pm 12.56^{* * * .9}$ & $<0.001$ \\
\hline $25.38 \pm 3.50$ & $27.96 \pm 4.03^{*}$ & $28.41 \pm 4.65$ & $31.44 \pm 6.87^{* * *}$ & $<0.001$ \\
\hline$+1.00 \pm 2.20$ & $+0.66 \pm 2.70$ & $+1.64 \pm 3.62$ & $+1.15 \pm 3.28$ & 0.646 \\
\hline $5(11) / 15(33)$ & $4(12) / 7(21)$ & $3(11) / 7(26)$ & $9(14) / 24(37)$ & 0.721 \\
\hline $3(1-14)$ & $11(7-33)$ & $5(4-18)$ & $17(7-27)^{* *}$ & 0.005 \\
\hline 0 & $2(1-5)$ & $0(0-0)$ & $3(2-6)$ & $<0.001$ \\
\hline $8(17.8)$ & $8(24.2)$ & $4(14.8)$ & 21 (32.3) & 0.186 \\
\hline $2(1-4)$ & $4(2-10)^{*}$ & $3(1-6)$ & $5(2-8)^{* *}$ & 0.003 \\
\hline $23(22-25)$ & $22(19-24)$ & 23 (19-25) & $21(19-23)^{* *}$ & 0.001 \\
\hline 33 (73.3) & 25 (75.8) & $22(81.5)$ & $52(80.0)$ & 0.805 \\
\hline $400(400-800)$ & $600(400-1000)$ & $1000(800-1000)^{*}$ & $1000(800-1500)^{* *,+}$ & $<0.001$ \\
\hline $12(26.7)$ & $13(40.6)$ & 7 (25.9) & $26(40.6)$ & 0.297 \\
\hline $92 \pm 13$ & $92 \pm 11$ & $88 \pm 15$ & $84 \pm 20$ & 0.037 \\
\hline $102 \pm 14$ & $101 \pm 10$ & $96 \pm 14$ & $93 \pm 18 *$ & 0.012 \\
\hline $0.74 \pm 0.07$ & $0.74 \pm 0.07$ & $0.74 \pm 0.07$ & $0.73 \pm 0.10$ & 0.428 \\
\hline $95 \pm 13$ & $95 \pm 10$ & $92 \pm 14$ & $87 \pm 20^{*}$ & 0.027 \\
\hline $101 \pm 15$ & $102 \pm 10$ & $97 \pm 14$ & $95 \pm 18$ & 0.060 \\
\hline $0.77 \pm 0.06$ & $0.76 \pm 0.06$ & $0.77 \pm 0.08$ & $0.74 \pm 0.11$ & 0.181 \\
\hline $3.3(2.6-4.2)$ & $3.3(2.5-3.9)$ & $4.0(2.6-5.1)$ & $4.4(3.4-5.1)^{* *,++}$ & $<0.001$ \\
\hline $0.19(0.12-0.28)$ & $0.13(0.06-0.28)$ & $0.18(0.11-0.31)$ & $0.15(0.10-0.25)$ & 0.245 \\
\hline $14(7-21)$ & $12(6-17)$ & $6(3-20)$ & $11(5-19)$ & 0.133 \\
\hline $69(29-186)$ & $45(21-151)$ & $76(30-134)$ & $49(24-173)$ & 0.618 \\
\hline
\end{tabular}

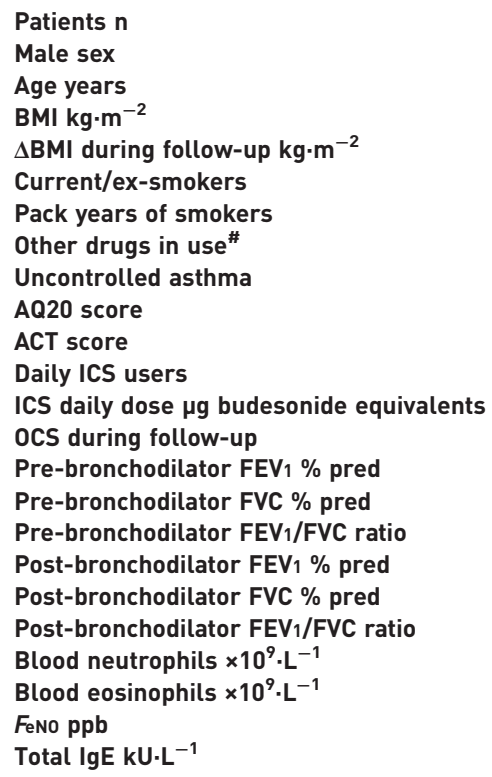

Data are presented as $\mathrm{n}(\%)$, mean \pm SD or median (interquartile range), unless otherwise stated. Systemic inflammation was defined as interleukin-6 $>1.55 \mathrm{pg} \cdot \mathrm{mL}^{-1}$ or high-sensitivity C-reactive protein $>4.12 \mathrm{mg} \cdot \mathrm{L}^{-1}$ or both. BMI: body mass index; AQ20: airways questionnaire $20 ; \mathrm{ACT}$ : asthma control test; ICS: inhaled corticosteroid; OCS: oral corticosteroid; FEV1: forced expiratory volume in $1 \mathrm{~s}$; FVC: forced vital capacity; FeNO: fraction of exhaled nitric oxide. ${ }^{\#}$ : number of drugs used for other indication than asthma/allergy. *: $p<0.05 ;{ }^{* *}$ : $p<0.01$; and ${ }^{* * *}$ : $p<0.001$ versus no comorbidity, no systemic inflammation. ${ }^{n}$ : $p<0.05$ versus no comorbidity, systemic inflammation. ${ }^{+}$: $p<0.05$; and ${ }^{++}$: $p<0.01$ versus $\geqslant 1$ comorbidity, no systemic inflammation.

Outcome of asthma in patients with or without systemic inflammation

To better characterise asthma in patients with systemic inflammation, we divided the cohort into two groups: 1) patients with low level of both hsCRP and IL-6 (no systemic inflammation); and 2) patients with elevated level of IL-6, hsCRP or both (systemic inflammation). Patients with systemic inflammation showed higher count of blood neutrophils, lower lung function, used higher ICS dose and had higher AQ20 score and lower ACT score compared with patients without systemic inflammation (table 3). Even after removal of all smokers from the analysis, the dose of ICS remained higher in patients with systemic inflammation (table S2).

In the whole patient cohort $(\mathrm{n}=170)$, Spearman's correlation coefficient between IL- 6 and hsCRP was $0.495(\mathrm{p}<0.001)$. Serum IL-6 level showed weak negative correlation to pre- and post-bronchodilator FEV1 (\% predicted) $(\rho=-0.218, p=0.006$ and $\rho=-0.244, p=0.001)$ and positive correlation to ICS dose ( $\rho=0.327, p<0.001)$. Serum IL- 6 showed no correlation to pre- $(\rho=-0.013, p=0.865)$ or post-bronchodilator FEV1/FVC $(\rho=-0.076, p=0.325)$.

\section{Outcome of asthma in patients with or without comorbidities}

To see the effect of comorbidity and multi-morbidity on asthma outcome, we divided the cohort into three groups: patients without comorbidities, with one comorbidity and with $\geqslant 2$ comorbidities (table 4 ). Patients with $\geqslant 2$ comorbidities showed poorer AQ20 and ACT scores, increased level of blood neutrophils and systemic inflammation in comparison to patients without comorbidities. Patients with one comorbidity or $\geqslant 2$ comorbidities differed from each other by age and use of drugs not related to treatment of asthma or allergy (table 4). Comorbidities in these patients are shown in table S3.

When adjusted for age, BMI, pack years and sex, having $\geqslant 2$ comorbidities was associated with lower ACT-score $(<21)$ (OR 2.477, CI 1.005-6.107; $\mathrm{p}=0.049$ ). The association between ACT score and $\geqslant 2$ comorbidity still showed borderline significance when adjusted for IL-6 in addition to age, pack-years, sex and BMI (OR 2.425, CI 0.979-6.005; p=0.056), suggesting that it may not be primarily mediated by IL-6. Having one comorbidity or level of IL- 6 were not predictors of poor ACT-score in adjusted analysis (data not shown). A negative correlation was found between the number of drugs to treat other diseases than allergy or asthma and ACT score (Spearman $\rho=-0.311, \mathrm{p}<0.001$ ). 
TABLE 3 Characteristics of patients with and without systemic inflammation

\begin{tabular}{|c|c|c|c|}
\hline & Without systemic inflammation & With systemic inflammation & p-value \\
\hline Patients n & 78 & 92 & \\
\hline Age years & $53.0 \pm 13.8$ & $60.7 \pm 13.1$ & $<0.001$ \\
\hline$\Delta \mathrm{BMI}$ during follow-up $\mathrm{kg} \cdot \mathrm{m}^{-2}$ & $+0.9 \pm 2.4$ & $+1.3 \pm 3.4$ & 0.330 \\
\hline Male sex & $29(37.2)$ & $34(37.0)$ & 0.999 \\
\hline Current/ex-smokers & $31(39.7)$ & $43(46.7)$ & 0.438 \\
\hline Other drugs in use ${ }^{\#}$ & $1(0-2)$ & $2(0-4)$ & $<0.001$ \\
\hline Blood neutrophils $\times 10^{9} \cdot \mathrm{L}^{-1}$ & $3.3(2.5-3.9)$ & $4.3(3.2-5.1)$ & $<0.001$ \\
\hline Blood eosinophils $\times 10^{9} \cdot \mathrm{L}^{-1}$ & $0.16(0.09-0.28)$ & $0.15(0.10-0.27)$ & 0.941 \\
\hline FenO ppb & $12(7-19)$ & $9(5-19)$ & 0.126 \\
\hline Total IgE kU. $\mathrm{L}^{-1}$ & $59(23-179)$ & $57(25-146)$ & 0.882 \\
\hline Pre-bronchodilator FEV $1 \%$ pred & $92 \pm 12$ & $85 \pm 19$ & 0.005 \\
\hline Uncontrolled asthma & $16(20.5)$ & $25(27.2)$ & 0.191 \\
\hline Daily ICS users & $58(74.4)$ & $74(80.4)$ & 0.362 \\
\hline ICS daily dose $\mu \mathrm{g}$ budesonide equivalents & $450(400-1000)$ & $1000(800-1425)$ & $<0.001$ \\
\hline$\geqslant 2$ OCS bursts in 2 years & $11(14.3)$ & $14(15.4)$ & 0.999 \\
\hline
\end{tabular}

To evaluate whether number of comorbidities associates with specific type of symptoms in patients with asthma, we investigated association between the number of comorbidities and specific questions in AQ20 and ACT. In adjusted analysis, patients with asthma and $\geqslant 2$ comorbidities had more often answered having breathlessness when walking upstairs and doing housework due to their chest trouble (Q12 and Q13 in AQ20) compared with patients without comorbidities (table 5).

Similarly, asthma kept from getting as much done in work, school or at home more often in patients with $\geqslant 2$ comorbidities (Q1 in ACT) (table 5).

For interest, in comparing the outcome of asthma in patients with $\geqslant 2$ comorbidities (without COPD) and patients with ACOS, we included the ACOS group for one analysis. In comparison with the ACOS group, patients with $\geqslant 2$ comorbidities were more often females and non-smokers, showed better lung function, had higher number of other than asthma- or allergy-related drugs in use (table 4) and had more often any psychiatric disease (table S3). Both groups showed systemic inflammation and elevated neutrophils, representing common features for ACOS and multi-morbidity without ACOS.

\section{Predictors of high ICS dose}

Higher use of ICS to treat asthma was evident in patients with systemic inflammation. We carried out multivariate logistic regression analyses to find out predictors of high ICS dose $(>800 \mu \mathrm{g}$ budesonide equivalent) at follow-up. Predictors of high ICS dose were IL-6 level $>1.55 \mathrm{pg} \cdot \mathrm{mL}^{-1}$, blood neutrophil level $>3.9 \times 10^{9} / \mathrm{L}$, blood eosinophils $>0.2 \times 10^{9} / \mathrm{L}, \mathrm{FEV}_{1} / \mathrm{FVC}$ post $<0.75$ at follow-up and $\mathrm{FEV} 1<80 \%$ predicted at diagnosis, while older age of asthma onset ( $>40$ years) was a borderline predictor (table 6 ). Comorbidity $(1$ or $\geqslant 2)$ was not a predictor of high ICS dose in the multivariate model and was removed from the model (data not shown). Predictors of medium ICS dose are shown in table S4.

\section{Discussion}

We showed that above half of patients with adult-onset asthma have systemic inflammation as defined by elevated level of IL-6, majority suffer from at least one comorbidity and one-third of several comorbidities. Elevated IL-6 was associated with higher ICS dose to treat asthma and multi-morbidity (two or more comorbidities in addition to asthma) associated with increased symptoms of asthma. The patients with both systemic inflammation and comorbidity had the poorest outcome of asthma. 
TABLE 4 Characteristics of patients with and without one or more comorbidities or asthma-COPD overlap syndrome (ACOS)

\begin{tabular}{|c|c|c|c|c|c|}
\hline & No comorbidities & 1 comorbidity & $\geqslant 2$ comorbidity & $\operatorname{ACOS}$ & p-value \\
\hline Age years & $51.33 \pm 14.22$ & $57.8 \pm 11.9 *$ & $64.9 \pm 11.5^{* * *,+}$ & $63.9 \pm 10.3^{* * *}$ & $<0.001$ \\
\hline$\Delta \mathrm{BMI}$ during follow-up $\mathrm{kg} \cdot \mathrm{m}^{-2}$ & $+1.2 \pm 2.8$ & $+1.4 \pm 3.0$ & $+0.6 \pm 3.1$ & $+1.0 \pm 3.1$ & 0.527 \\
\hline Male sex & 27 (37.5) & $19(40.4)$ & 17 (33.3) & $20(66.7)^{*, 9}$ & 0.021 \\
\hline Current/ex-smokers & $20(41.7)$ & $23(48.9)$ & $21(41.2)$ & $30(100)^{* * *,+++, \text { ๆศๆ }}$ & $<0.001$ \\
\hline Blood neutrophils $\times 10^{9} \cdot \mathrm{L}^{-1}$ & $3.5(2.6-4.7)$ & $3.6(2.8-4.4)$ & $4.2(3.1-5.0)^{*,+}$ & $4.3(3.5-5.5)^{* *,+}$ & 0.007 \\
\hline Blood eosinophils $\times 10^{9} \cdot \mathrm{L}^{-1}$ & $0.18(0.12-0.28)$ & $0.15(0.08-0.26)$ & $0.14(0.08-0.27)$ & $0.20(0.11-0.29)$ & 0.216 \\
\hline FenO ppb & $12(5-20)$ & $11.5(7-19)$ & $10(5-18)$ & $8.5(5-15)$ & 0.312 \\
\hline Total IgE kU: $\mathrm{L}^{-1}$ & $71(30-165)$ & $47(21-111)$ & $52(24-204)$ & $62(20-165)$ & 0.615 \\
\hline Pre-bronchodilator FEV $1 \%$ pred & $90 \pm 14$ & $87 \pm 20$ & $87 \pm 15$ & 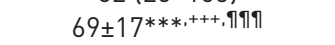 & $<0.001$ \\
\hline Post-bronchodilator FEV $1 \%$ pred & $94 \pm 13$ & $90 \pm 20$ & $90 \pm 15$ & $73 \pm 17 * * *,+++$, ๆๆๆ & $<0.001$ \\
\hline ICS daily dose $\mu \mathrm{g}$ budesonide equivalents & $775(400-1000)$ & $800(400-1000)$ & $800(500-1500)$ & $800(800-1200)$ & 0.162 \\
\hline$\geqslant 2$ ocs bursts in 2 years & $7(9.7)$ & $9(20.0)$ & $9(17.6)$ & $3(10.3)$ & 0.351 \\
\hline Other drugs in use ${ }^{\#}$ & 0 & $2(1-2)^{* * *}$ & $5(4-7) * * *,+++$ & 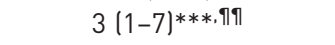 & $<0.001$ \\
\hline hsCRP $\mathrm{mg} \cdot \mathrm{L}^{-1}$ & $0.98(0.36-1.70)$ & $1.47(0.49-2.79)$ & $1.45(0.81-4.15)^{* *}$ & $0.96(0.67-3.04)$ & 0.027 \\
\hline $\mathrm{IL}-6 \mathrm{pg} \cdot \mathrm{mL}^{-1}$ & $1.23(0.89-1.96)$ & $1.72(1.22-2.91)^{* *}$ & $2.27(1.45-4.84)^{* * *}$ & $3.17(1.85-5.24)^{* * *,+}$ & $<0.001$ \\
\hline
\end{tabular}

Data are presented as $\mathrm{n}(\%)$, mean \pm SD or median (interquartile range), unless otherwise stated. BMI: body mass index; FeNO: fraction of exhaled nitric oxide; FEV1: forced expiratory volume in $1 \mathrm{~s}$; FVC: forced vital capacity; AQ20: airways questionnaire 20; ACT: asthma control test; ICS: inhaled corticosteroid; OCS: oral corticosteroid; hSCRP: high-sensitivity C-reactive protein; IL: interleukin. \#: number of drugs used for other indication than asthma/allergy. ${ }^{*}: p<0.05 ;{ }^{* *}: p<0.01$; and ${ }^{* * *}: p<0.001$ versus no comorbidities. ${ }^{+}: p<0.05 ;{ }^{++}: p<0.01 ;$ and ${ }^{+++}: p<0.001$

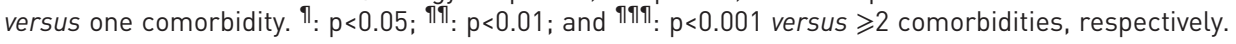

In our study, above half of patients with adult-onset asthma had elevated level of IL-6, indicative of systemic inflammation. As there exists no widely accepted limits for the presence of systemic inflammation, our definition was based on upper quartiles of IL-6 and hsCRP reported in healthy subjects [15]. Our study suggests that hsCRP is not linked to lung function in asthmatic patients, as was also proposed by a previous study [15]. IL-6 has been associated with reduced lung function in patients with asthma $[15,24]$ and may be a more significant biomarker of systemic inflammation associated with outcome of asthma. In concordance with the previous studies, we found decreased lung function and elevated neutrophils in patients with elevated IL-6, as well as negative correlation between IL-6 and FEV1 [15, 24].

IL-6 is a pleiotropic cytokine [25] being produced by multiple cells including inflammatory cells and airway epithelial cells upon damage or activation. It has been previously considered as a non-specific marker of inflammation in a variety of diseases, even though large body of evidence suggests a more functional role. Elevated IL- 6 has been regarded as a marker of frailty in older age and is associated with increased risk of mortality and morbidity [26]. Of the various mechanisms it may contribute to asthma, best characterised is its stimulatory effect on Th2 differentiation and IL-13 production [27]. In support of this pathway being operational, in younger adults with mild-to-moderate allergic asthma, IL-6 and IL-13 were correlated [28]. IL- 6 has also been reported to stimulate Th17 differentiation in the presence of transforming growth factor (TGF)- $\beta$ suggesting that IL-6 may promote Th17-mediated neutrophil-predominant severe asthma $[27,28]$. In addition, via activating TGF- $\beta$ [29], IL-6 is able to induce fibrosis, airway remodelling and loss of post-bronchodilator lung function [28]. Elevated blood neutrophils in our patients with systemic inflammation support involvement of Th17 rather than Th2 pathway. For further support, Th17 cells have been reported as steroid-resistant [30], which could account for the higher ICS dose used by the patients with elevated IL-6. However, we cannot rule out the possibility that ICS treatment has resulted in reduced eosinophilia but increased neutrophilia in our patients as has been suggested by studies showing promotion of neutrophil survival by ICS [31-33]. In normal physiological situation glucocorticoids reduce production of IL-6 but its high levels in patients with adult-onset asthma suggests that this capacity of glucocorticoids may be disturbed. For example chronic psychological stress has been shown to reduce capacity of glucocorticoids to suppress production of IL-6 [34]. 
TABLE 5 Specific questions in airways questionnaire 20 (AQ20) and asthma control test in patients with or without comorbidities

$\begin{array}{cccc}\text { No } & 1 & \geqslant 2 & \text { Unadjusted } \\ \text { comorbidities } & \text { comorbidity } & \text { comorbidity } & \begin{array}{c}\text { overall } \\ \text { p-value }\end{array}\end{array}$

\section{Patients $\mathrm{n}$ \\ AQ20; $n(\%)$ answering "yes"}

Q1. Do you suffer from coughing attacks during the day?

Q2. Because of your chest trouble do you often feel restless?

Q3. Because of your chest trouble do you feel breathless when maintaining the garden?

Q4. Do you worry when going to a friend's house that there might be something there that will set off an attack of chest trouble?

Q5. Do you suffer from chest symptoms as a result of exposure to strong smells, cigarette smoke or perfume?

Q6. Is your partner bothered by your chest problems?

Q7. Do you feel breathless while trying to sleep?

Q8. Do you worry about the long term effects on your health of the drugs that you have to take because of your chest trouble?

Q9. Does getting emotionally upset make your chest trouble worse?

Q10. Because of your chest trouble are there times when you have difficulty getting around the house?

Q11. Because of your chest trouble do you suffer from breathlessness carrying out activities at work?

Q12. Do you feel breathless walking upstairs because of your chest trouble?

Q13. Because of your chest trouble do you suffer from breathlessness doing housework?

Q14. Because of your chest trouble do you go home sooner than others after a night out?

Q15. Because of your chest trouble do you suffer from breathlessness when you laugh?

Q16. Because of your chest trouble do you often feel impatient?

Q17. Because of your chest trouble do you feel that you cannot enjoy a full life?

Q18. Do you feel drained after a cold because of your chest trouble?

Q19. Do you have a feeling of chest heaviness?

Q20. Do you bother much about your chest trouble?

\section{Asthma control test; $\mathbf{n}(\%)$ scoring $\leqslant 3$}

Q1. Asthma kept the patient from getting as much done at work, school or at home all, most or some of the time

Q2. Patient had shortness of breath at least 3-6 times/week

Q3. Asthma symptoms woke the patients up at night or earlier than usual in the morning at least once a week

Q4. The patient has used rescue inhaler or nebuliser medication at least 2-3 times per week

Q5. Patient rated his/her asthma as somewhat controlled, poorly controlled or not at all contolled

72

$$
\begin{array}{r}
28 \\
3 \\
8 \\
19
\end{array}
$$

$3(4.2)$

$6(8.3)$

16 (22.9)

$15(21.4)$

0

7 (10)

13 (18.3)

2 (2.8)

1 (1.5)

$11(15.7)$

6 (8.6)

4 (5.7)

23 (34.3)

$14(19.7)$

6 (8.6)

$7(9.7)$

7 (9.7)

8 (11.2)

14 (19.5)

$8(11.1)$
47

$18(41.9)$
$13(30.2)$
$8(18.2)$

17 (39.5)

$34(75.6)$

6 (13.6)

11 (25)

16 (36.4)

16 (35.6)

5 (11.6)

$9(20.6)$

16 (36.4)

5 (11.4)

7 (17.1)

$6(13.6)$

9 (20.5)

$14(32.6)$

21 (47.7)

14 (31.1)

10 (22.2)

$$
9 \text { (20) }
$$

8 (17.8)

8 (17.7)

15 (33.3)

$14(31.1)$
51

$29(56.9)$
$7(14.0)$
$18(36)$

11 (21.6)

40 (76.9)

0.534

$6(11.8)$

$6(11.5)$

9 (18.4)

0.166

0.044

0.115

0.140

0.001

0.004

$\mathbf{0 . 0 0 3 / 0 . 3 2 4}$

$0.439 / 0.247$

0.156

0.261

0.008

ND

0.100

$<0.001$

$0.482 / 0.015$

29 (60.4)

$<0.001$

$0.306 / 0.007$

0.013

$\mathbf{0 . 0 2 3 / 0 . 1 4 6}$

4 (9.3)

11 (22.4)

0.484

5 (10.2)

0.148

9 (18.4)

0.001

$0.002 / 0.090$

25 (51)

0.154

21 (40.4)

10 (19.2)

0.042

0.098

$0.383 / 0.172$

15 (28.8)

0.024

$0.066 / \mathbf{0 . 0 3 3}$

14 (26.9)

0.043

0.578

$0.431 / 0.148$

8 (15.4)

0.147

17 (32.6)

0.010

\footnotetext{
\#: adjusted for body mass index, age, pack-years and sex. The first adjusted p-value shown is for comparison between groups of 1 comorbidity
} versus no comorbidities and the second for comparison between $\geqslant 2$ comorbidities versus no comorbidities. ND: not determined.

Because patients with systemic inflammation used higher dose of ICS in unadjusted analysis, we constructed a multivariate model to elucidate predictors of high ICS dose. To our knowledge, this is the first study to identify predictors of high ICS dose in a follow-up study of adult asthma. Our results suggests that the patients with post-bronchodilator $\mathrm{FEV}_{1}<80 \%$ at diagnosis (mostly untreated) were users of medium to high ICS dose 12 years after diagnosis. In support of this, in a follow-up study, lower FEV 1 


\begin{tabular}{|c|c|c|c|}
\hline Variable $^{+}$ & OR & $95 \% \mathrm{Cl}$ & $p$-value \\
\hline Age at onset $>40$ years & 3.28 & $0.97-11.04$ & 0.056 \\
\hline Male sex & 0.47 & $0.14-1.62$ & 0.233 \\
\hline FEV1 post-bronchodilator at diagnosis $<80 \%$ pred & 4.50 & $1.01-19.98$ & 0.048 \\
\hline Waist circumference $>80 / 94 \mathrm{~cm}$ (female $/$ male) at follow-up & 0.48 & $0.12-1.98$ & 0.312 \\
\hline FEV $1 / F V C$ post $<0.75$ at follow-up & 3.84 & $1.29-11.40$ & 0.015 \\
\hline Every 10 pack-years smoked at follow-up & 1.34 & $0.68-2.64$ & 0.396 \\
\hline $\mathrm{IL}-6>1.55 \mathrm{pg} \cdot \mathrm{mL}^{-1}$ at follow-up & 3.49 & $1.08-11.29$ & 0.037 \\
\hline Blood neutrophils $>3.9 \times 10^{9} \cdot \mathrm{L}^{-1}$ at follow-up & 5.91 & $1.85-18.89$ & 0.003 \\
\hline Blood eosinophils $>0.2 \times 10^{9} \cdot \mathrm{L}^{-1}$ at follow-up & 7.25 & $2.25-23.40$ & 0.001 \\
\hline \multicolumn{4}{|l|}{ ACT score at follow-up } \\
\hline $16-20$ & 2.12 & $0.55-8.18$ & 0.275 \\
\hline $5-15$ & 3.18 & $0.50-20.92$ & 0.221 \\
\hline
\end{tabular}

FEV1: forced expiratory volume in $1 \mathrm{~s} ;$ FVC: forced vital capacity; ACT: asthma control test. Bold indicates significance. ${ }^{\#}$ : $>800 \mu \mathrm{g}$ daily dose of budesonide equivalent. ": versus patients with $0-400 \mu \mathrm{g}$ budesonide equivalent in use. ${ }^{+}$: all factors adjusted for in the final model are shown; also other factors, such as body mass index were tested but excluded from the final analysis because of lower predictive value compared with the factors included (e.g. body mass index versus waist circumference).

within 1 year from diagnosis was found to predict more severe asthma 5.8 years later in adult-onset asthma [35]. Also increased bronchial obstruction at follow-up $(<0.75)$ predicted a high dose of ICS. In agreement, in a recent cluster analysis of patients with and without fixed airflow obstruction, the patients with persistent obstruction were often users of high ICS [36]. In addition, patients with elevated markers of inflammation in their blood (eosinophils $>0.2 \times 10^{9} / \mathrm{L}$ and neutrophils $>3.9 \times 10^{9} / \mathrm{L}$ ) despite of anti-inflammatory treatment were more likely users of high ICS dose, suggesting more steroid-resistant inflammation. Obesity has been previously associated with steroid resistance, high-dose ICS and use of several controllers but, in adjusted analysis, neither BMI nor waist circumference explained high ICS dose. IL-6 is elevated in obese subjects but functions beyond obesity and may present a more precise marker of the systemic inflammation and/or metabolic changes that affect treatment and outcome of asthma compared with obesity. Surprisingly, smoked pack-years $>10$ did not predict use of high ICS dose. ICS has been proven less effective in smoking patients with asthma [37] which may have led to preferring combination treatment with lower ICS and long-acting $\beta_{2}$-agonist instead of high ICS. According to our result, bronchial obstruction that is often developed to smokers is a better predictor of high ICS dose than smoking. Altogether, lung function and inflammatory parameters including elevated IL-6 were found to associate with high dose ICS therapy.

In most studies with asthma, patients having significant comorbidities are excluded. SAAS is a real-life follow-up study where patients with comorbid conditions are included. As no standard approach is available to measure comorbidity or multi-morbidity, we utilised criteria used in a large evaluation of medical practice patients to ensure wide engagement of comorbid conditions [23]. In this cohort with carefully characterised patients with adult-onset asthma, 58\% of the patients suffered from one comorbidity and 30\% suffered from several comorbidities. The most prevalent comorbidities were hypertension, any psychiatric disease and type 2 diabetes. In another study of patients with asthma from general practice (aged $40-75$ years), 53\% were reported to suffer from at least one comorbidity, and $20 \%$ of $\geqslant 2$ comorbidities [8]. Another study including only patients with adult-onset asthma (mean age 52 years) found $50.5 \%$ prevalence of comorbid conditions but e.g. rhinitis and sinusitis were included as comorbid conditions in contrast to our study [38]. Our study, together with the previous ones supports the view that comorbidities and multi-morbidity are common in adult or late-onset asthma and confirms the recent population-based studies [6, 39, 40], indicating a high burden of comorbidities in patients with asthma.

We found that patients with at least two comorbidities showed increased symptoms of asthma as determined by poorer score in AQ20 and ACT compared with patients without comorbidities. Increased symptoms in multi-morbid patients were mostly related to breathlessness during daily activities. Obesity has been shown to lower asthma control and complicate asthma [41] but the effect of multi-morbidity on symptoms was independent from BMI. According to our data, IL-6 may partially account for the aggravated symptoms but because of involvement of several comorbidities, it is more likely explained by a multifactorial mechanism including variety of mediators [7]. Mechanisms may include overspill of inflammation as well as stress of multi-morbidity. Multi-morbid patients showed elevated blood 
neutrophils but whether neutrophilic airway inflammation explains the higher symptoms remains obscure. Accumulation of several diseases may also end up in worsening patients' perception of symptoms. Our results are well in line with the results of the population-based studies $[6,39,40]$ showing that asthmatic patients with comorbidities have increased number of health claims and unscheduled health care visit. Logically, asthma with comorbidities has been shown to result in decreased asthma-related and general quality of life [8], and this was most pronounced in patients with many comorbidities. Of the specific comorbidities, hypertension ( $72 \%$ in our multi-morbid group) has been linked to slightly increased use of short-acting $\beta_{2}$-agonist, more frequent emergency department visit/hospitalisation and oral corticosteroid dispensings in patients with asthma [42]. Our patients with comorbidities were using on average three drugs for treatment of their comorbidities, and the possibility remains that polypharmacy or use of certain drugs is responsible for the effects seen rather than comorbidities. In support of this, in a geriatric Korean population, fewer number of medication for comorbidities but not fewer number of comorbidities predicted improved asthma control after 6 months of follow-up [43].

Given the recent interest on ACOS, we compared multi-morbid asthma patients (without ACOS) to patients with ACOS. The only difference found in parameters related to asthma outcome was poorer lung function in patients with ACOS, being in line with a previous study [44], which however lacked comparison between asthma control and symptoms. Higher level of IL-6 and sputum neutrophils in ACOS was also previously reported, with similar comorbidity index between the groups [44]. In our study, systemic inflammation and neutrophils were similarly elevated in both ACOS and patients with $\geqslant 2$ comorbidities, which may be at least partially explained by the higher load of comorbidities, and especially psychiatric diseases in the latter group. Psychiatric diseases have been associated with systemic inflammation, worse asthma control and asthma-related quality of life $[9,11]$.

Altogether, we showed that comorbidities and elevated IL-6 are common among patients with adult-onset asthma and are associated with worse outcome of asthma. Whether the origin of IL-6 in patients with adult-onset asthma is obesity, smoking, stress, asthma or another chronic disease is not revealed by our data but plenty of evidence suggests that elevated levels of IL-6 contribute to development of multi-morbidity [26], and participate in pathophysiology of asthma [27, 28]. Our findings add by suggesting that IL-6 also complicates treatment of asthma. Multi-morbid patients with asthma were more symptomatic when compared to asthma without comorbidities, a phenomenon that is proposed to be multifactorial rather than mediated solely by IL- 6 . In clinics, IL- 6 could be useful in identifying patients with poor response to ICS treatment, and in identifying those patients, who could benefit from life-style changes as part of their treatment programme, as exercise has been shown to reduce systemic inflammation and improve outcome of asthma [45]. Additionally, anti-IL-6 therapies deserve to be investigated as an alternative treatment strategy.

\section{Acknowledgements}

Aino Sepponen (Dept of Respiratory Medicine, Seinäjoki Central Hospital, Seinäjoki, Finland) is gratefully acknowledged for her help through all the stages of this work.

\section{References}

1 Bisgaard H, Bønnelykke K. Long-term studies of the natural history of asthma in childhood. J Allergy Clin Immunol 2010; 126: 187-197.

2 Ilmarinen P, Tuomisto LE, Kankaanranta H. Phenotypes, risk factors and mechanisms of adult-onset asthma. Mediators Inflamm 2015; 2015: 1-19.

Amelink M, de Nijs SB, de Groot JC, et al. Three phenotypes of adult-onset asthma. Allergy 2013; 68: 674-680. Wenzel SE. Asthma phenotypes: the evolution from clinical to molecular approaches. Nat Med 2012; 18: 716-725. Tuomisto LE, Ilmarinen P, Kankaanranta H. Prognosis of new-onset asthma diagnosed at adult age. Respir Med 2015; 109: 944-954.

6 Gershon AS, Wang C, Guan J, et al. Burden of comorbidity in individuals with asthma. Thorax 2010; 65: $612-618$.

7 Kankaanranta H, Kauppi P, Tuomisto LE, et al. Emerging comorbidities in adult asthma: risks, clinical associations and mechanisms. Mediators Inflamm 2016; 2016: 3690628.

8 Wijnhoven HA, Kriegsman DM, Hesselink AE, et al. The influence of comorbidity on health-related quality of life in asthma and COPD patients. Respir Med 2003; 97: 468-475.

9 Lavoie KL, Bacon SL, Barone S, et al. What is worse for asthma control and quality of life: depressive disorders, anxiety disorders, or both? Chest 2006; 130: 1039-1047.

10 Kapadia SG, Wei C, Bartlett SJ, et al. Obesity and symptoms of depression contribute independently to the poor asthma control of obesity. Respir Med 2014; 108: 1100-1107.

11 Jiang M, Qin P, Yang X. Comorbidity between depression and asthma via immune-inflammatory pathways: a meta-analysis. J Affect Disord 2014; 166: 22-29.

12 Esser N, Legrand-Poels S, Piette J, et al. Inflammation as a link between obesity, metabolic syndrome and type 2 diabetes. Diabetes Res Clin Pract 2014; 105: 141-150.

13 Skeoch S, Bruce IN. Atherosclerosis in rheumatoid arthritis: is it all about inflammation? Nat Rev Rheumatol 2015; 11: 390-400.

14 Slavich GM, Irwin MR. From stress to inflammation and major depressive disorder: a social signal transduction theory of depression. Psychol Bull 2014; 140: 774-815. 
15 Wood LG, Baines $\mathrm{KJ}$, Fu J, et al. The neutrophilic inflammatory phenotype is associated with systemic inflammation in asthma. Chest 2012; 142: 86-93.

$16 \mathrm{Fu} \mathrm{JJ}$, Baines KJ, Wood LG, et al. Systemic inflammation is associated with differential gene expression and airway neutrophilia in asthma. OMICS 2013; 17: 187-199.

17 Kankaanranta H, Ilmarinen P, Kankaanranta T, et al. Seinajoki Adult Asthma Study (SAAS): a protocol for a 12-year real-life follow-up study of new-onset asthma diagnosed at adult age and treated in primary and specialised care. NPJ Prim Care Respir Med 2015; 25: 15042.

18 Global Initiative for Asthma (GINA), Global Initiative for Chronic Obstructive Lung Disease (GOLD). Asthma, COPD and Asthma-COPD Overlap Syndrome (ACOS). www.goldcopd.org/ Date last accessed: April, 2015. Date last updated: April, 2015.

19 Miller MR, Hankinson J, Brusasco V, et al. Standardisation of spirometry. Eur Respir J 2005; 26: 319-338.

20 American Thoracic Society, European Respiratory Society. ATS/ERS recommendations for standardized procedures for the online and offline measurement of exhaled lower respiratory nitric oxide and nasal nitric oxide, 2005. Am J Respir Crit Care Med 2005; 171: 912-930.

21 Barley EA, Quirk FH, Jones PW. Asthma health status measurement in clinical practice: validity of a new short and simple instrument. Respir Med 1998; 92: 1207-1214.

22 Global Initiative for Asthma (GINA). Global Strategy for Asthma Management and Prevention. www.ginasthma. org/ Date last updated: 2010.

23 Barnett K, Mercer SW, Norbury M, et al. Epidemiology of multimorbidity and implications for health care, research, and medical education: a cross-sectional study. Lancet 2012; 380: 37-43.

24 Morjaria JB, Babu KS, Vijayanand P, et al. Sputum IL-6 concentrations in severe asthma and its relationship with FEV1. Thorax 2011; 66: 537.

25 Hunter CA, Jones SA. IL-6 as a keystone cytokine in health and disease. Nat Immunol 2015; 16: 448-457.

26 Michaud M, Balardy L, Moulis G, et al. Proinflammatory cytokines, aging, and age-related diseases. J Am Med Dir Assoc 2013; 14: 877-882.

27 Rincon M, Irvin CG. Role of IL-6 in asthma and other inflammatory pulmonary diseases. Int J Biol Sci 2012; 8: 1281-1290.

28 Neveu WA, Allard JL, Raymond DM, et al. Elevation of IL-6 in the allergic asthmatic airway is independent of inflammation but associates with loss of central airway function. Respir Res 2010; 11: 28. 9921-11-28.

29 Zhang XL, Topley N, Ito T, et al. Interleukin-6 regulation of transforming growth factor (TGF)-beta receptor compartmentalization and turnover enhances TGF-betal signaling. J Biol Chem 2005; 280: 12239-12245.

30 McKinley L, Alcorn JF, Peterson A, et al. TH17 cells mediate steroid-resistant airway inflammation and airway hyperresponsiveness in mice. J Immunol 2008; 181: 4089-4097.

31 Perttunen $\mathrm{H}$, Moilanen E, Zhang X, et al. Beta2-agonists potentiate corticosteroid-induced neutrophil survival. COPD 2008; 5: 163-169.

32 Zhang X, Moilanen E, Adcock IM, et al. Divergent effect of mometasone on human eosinophil and neutrophil apoptosis. Life Sci 2002; 71: 1523-1534.

33 Ilmarinen P, Kankaanranta H. Eosinophil apoptosis as a therapeutic target in allergic asthma. Basic Clin Pharmacol Toxicol 2014; 114: 109-117.

34 Miller GE, Cohen S, Ritchey AK. Chronic psychological stress and the regulation of pro-inflammatory cytokines: a glucocorticoid-resistance model. Health Psychol 2002; 21: 531-541.

35 Rönmark E, Lindberg A, Watson L, et al. Outcome and severity of adult onset asthma: report from the obstructive lung disease in northern Sweden studies (OLIN). Respir Med 2007; 101: 2370-2377.

36 Konstantellou E, Papaioannou AI, Loukides S, et al. Persistent airflow obstruction in patients with asthma: characteristics of a distinct clinical phenotype. Respir Med 2015; 109: 1404-1409.

37 Adcock IM, Marwick J, Casolari P, et al. Mechanisms of corticosteroid resistance in severe asthma and chronic obstructive pulmonary disease (COPD). Curr Pharm Des 2010; 16: 3554-3573.

38 Sözener ZÇ, Aydin Ö, Mungan D, et al. Prognosis of adult asthma: a 7-year follow-up study. Ann Allergy Asthma Immunol 2015; 114: 370-373.

39 Gershon AS, Guan J, Wang C, et al. Describing and quantifying asthma comorbidity: a population study. PLoS One 2012; 7: e34967.

40 Steppuhn H, Langen U, Keil T, et al. Chronic disease comorbidity of asthma and unscheduled asthma care among adults: results of the national telephone health interview survey German Health Update (GEDA) 2009 and 2010. Prim Care Respir J 2014; 23: 22-29.

41 Lessard A, Turcotte H, Cormier Y, et al. Obesity and asthma: a specific phenotype? Chest 2008; 134: 317-323.

42 Christiansen SC, Schatz M, Yang SJ, et al. Hypertension and Asthma: A Comorbid Relationship. J Allergy Clin Immunol Pract 2016; 4: 76-81.

43 Ban GY, Ye YM, Lee Y, et al. Predictors of asthma control by stepwise treatment in elderly asthmatic patients. J Korean Med Sci 2015; 30: 1042-1047.

44 Fu JJ, McDonald VM, Gibson PG, et al. Systemic Inflammation in Older Adults With Asthma-COPD Overlap Syndrome. Allergy Asthma Immunol Res 2014; 6: 316-324.

45 França-Pinto A, Mendes FA, de Carvalho-Pinto RM, et al. Aerobic training decreases bronchial hyperresponsiveness and systemic inflammation in patients with moderate or severe asthma: a randomised controlled trial. Thorax 2015; 70: 732-739. 